\begin{tabular}{|c|l|}
\hline Title & Single photon emission from InA sP quantum dots embedded in density-controlled InP nanowires \\
\hline Author(s) & Shougo, Y anase; Hirotaka, Sasakura; Shinjiro, Hara; Junichi, Motohisa \\
\hline Citation & $\begin{array}{l}\text { Japanese Journal of A pplied Physics, 56/4S), O4CPO4 } \\
\text { https://doi.org/40.7567/JJA.56.04CPO4 }\end{array}$ \\
\hline Issue Date & 2017-02-17 \\
\hline Doc URL & http://hdl.handle.net/2115/68330 \\
\hline Rights & @2017 The Japan Society of A pplied Physics \\
\hline Type & article (author version) \\
\hline File Information & SS16173.pdf \\
\hline
\end{tabular}

Instructions for use 


\title{
Single-photon emission from InAsP quantum dots embedded in density-controlled InP nanowires
}

\author{
Shougo Yanase ${ }^{1}$, Hirotaka Sasakura ${ }^{2}$, Shinjiro Hara ${ }^{1,3}$, and Junichi Motohisa*1,3 \\ ${ }^{1}$ Graduate School of Information Science and Technology, Hokkaido University, Sapporo 060-0814, Japan \\ ${ }^{2}$ Graduate School of Engineering, Hokkaido University, Sapporo 060-8628, Japan \\ ${ }^{3}$ Research Center for Integrated Quantum Electronics, Hokkaido University, Sapporo 060-8628, Japan
}

\begin{abstract}
We attempted to control the density and size of InP-based nanowires (NWs) and nanowire quantum dots (NW-QDs) during selective-area metalorganic vapor phase epitaxy. $\ln P$ nanowire arrays with a $5 \mu \mathrm{m}$ pitch and an average NW diameter $d$ of $67 \mathrm{~nm}$ were successfully grown by optimization of growth conditions. InAsP quantum dots were embedded in these density-controlled InP NW arrays, and clear single-photon emission and exciton-biexciton cascaded emission were confirmed by excitation-dependent photoluminescence and photon correlation measurements.
\end{abstract}

\section{Introduction}

Semiconductor nanowires (NWs) and quantum dots (QDs) are important nanomaterials with various applications, particularly in the field of micro- and nanoelectronics, owing to their unique forms and excellent electronic/optical properties. The combination of these two materials, which is referred to as NW-QDs and can easily be realized by embedding QDs inside NWs during growth, is expected to provide additional unique features utilizing the advantages of both QDs and NWs. ${ }^{1-9)}$ For example, the advantages of NW-QDs as compared with those of conventional self-assembled QDs (SA-QDs) formed in the Stranski-Krastanov growth mode are as follows. First, QDs are formed at predetermined positions by a combination of lithography and epitaxial growth. This also enables the control of the density of NW and NW-QD arrays. The second advantage is the easy fabrication of electrical contacts on NWs, and the resulting low-density NW-QD arrays are beneficial for avoiding complicated postgrowth selection or processing for their application to NW-based light-emitting diodes. ${ }^{3,10}$ ) Third, light extraction from QDs is more efficient than that from QDs embedded in a planar structure with a high refractive index, while it still enables the formation of a cavity structure and can be utilized to control spontaneous emission. ${ }^{11)}$ Fourth, NWs are generally formed

*E-mail: motohisa@ist.hokudai.ac.jp 
along the [111] direction of zincblende crystals in III-V semiconductors, and the higher symmetry of QDs on the (111)-oriented surface than those on the (100)-oriented surface makes it possible to suppress the fine-structure splitting of excitons in QDs. ${ }^{12)}$ Note that attempts have been made to realize all these features in SA-QD systems. As reported in Refs. 13 and 14 , postgrowth selection followed by processing has been realized by sophisticated in situ lithography with SA-QDs embedded in microcavities and $p n$ junctions. In NW-QD systems, on the other hand, these features, which are particularly important for single-photon sources, are realized in a more natural and straightforward way.

Among III-V semiconductor NWs, InP-based NWs ${ }^{15-21)}$ are important for photonic devices because their band-gap energy is compatible with the wavelength used in optical fiber telecommunication, and one of the important applications of QDs is in single-photon sources used in quantum information and quantum cryptography. The formation of NW-QDs by InPbased materials by a catalyst-assisted vapor-liquid-solid (VLS) mechanism ${ }^{22,23)}$ or selectivearea metalorganic vapor phase epitaxy (SA-MOVPE) ${ }^{5,24)}$ has been studied towards achieving a single-photon emitter in the optical communication band. Detailed studies have been carried out on their optical properties ${ }^{25)}$ and single-photon emission ${ }^{23,24)}$ and entangled photon emission $^{26)}$ has been demonstrated in these studies. Some of the remaining issues of NW-QDbased single-photon emitters are the control of emission wavelength and electrically driven operation. For the former, the capability of emission control by changing the compositions of As and P or the growth time of InAsP was partly demonstrated in our previous study, 5) but to the best of our knowledge, this has not yet been achieved in the optical communication band and remains a challenge. For the latter, as mentioned in the previous paragraph, NWQDs have a major advantage for electrical operation; thus, if the NWs are sufficiently apart from each other, one can easily realize a single-photon emitter without postgrowth selection. For this, however, optimization of growth conditions for low-density NWs is necessary because the distance between the NWs critically affects the dynamics of their growth via the gas phase/surface diffusion of growth species, for example, as reported in Ref. 27. In addition, smaller and low-density arrays of patterned mask holes in SA-MOVPE sometimes result in a difficulty in the initial nucleation of NWs. ${ }^{28)}$ It is thus necessary to realize NW arrays with a small NW diameter $(<100 \mathrm{~nm})$ independent of the NW pitch $a$, which is important for ensuring the formation of a single QD in a single NW with good wavelength reproducibility.

The purpose of this study is to control the density as well as the size of InP NWs and realize NW-QDs using density-controlled NW arrays. We will first show that the conventional growth conditions are not directly applicable, but by optimizing the growth conditions, 
InP NW arrays with $5 \mu \mathrm{m}$ pitch and an average NW diameter of $67 \mathrm{~nm}$ were successfully formed. Using these optimized growth conditions, a single InAsP QD was also realized in a low-density InP NW array. The grown NW-QDs showed clear excitonic and biexcitonic photoluminescence (PL). Furthermore, photon correlation measurement revealed clear photon antibunching and bunching emissions in exciton and biexciton emissions, respectively, which is the most unambiguous proof and important property of single-photon emitters with QDs.

\section{Experimental procedure}

The selective-area growth of InP-based NWs was carried out by MOVPE on an InP (111)A substrate, on which a periodic array of circular mask holes with the diameter $d_{0}$ was defined. The mask holes were arranged in a triangular lattice with the period $a$, ranging from 1 to 6 $\mu \mathrm{m}$. The hole size of the $\mathrm{SiO}_{2}$ mask was in the range from 50 to $100 \mathrm{~nm}$. Trimethylindium (TMIn), tertiarybutylphosphine (TBP), and arsine $\left(\mathrm{AsH}_{3}\right)$ were used as source materials. In this experiment, the partial pressure of TBP [TBP] and that of $\mathrm{AsH}_{3}\left(\left[\mathrm{AsH}_{3}\right]\right.$ ) were fixed at $9.35 \times 10^{-5}$ and $5.33 \times 10^{-6}$ atm, respectively. The partial pressure of TMIn ([TMIn]) was varied to give a V/III ratio in the range from 38.6 to 89.9. The growth temperature $T_{\mathrm{G}}$ of InP NWs was fixed at $605{ }^{\circ} \mathrm{C}$. The V/III ratio was slightly higher and $T_{\mathrm{G}}$ was lower than those used in our previous study ${ }^{20)}$ on optimizing InP NWs with the wurtzite crystal structure. The effect of this difference will be discussed later.

After the optimization of the InP NW growth conditions, InAsP QDs were formed. The growth started with the $10 \mathrm{~min}$ growth of InP at $585{ }^{\circ} \mathrm{C}$. Then, the growth temperature was lowered to $580{ }^{\circ} \mathrm{C}$ (which took about $3 \mathrm{~min}$ ) and InAsP was grown for $3 \mathrm{~s}$ by supplying TMIn, TBP, and $\mathrm{AsH}_{3}$ simultaneously. Then, without interruption, the $\mathrm{AsH}_{3}$ supply was switched off to grow InP for $2 \mathrm{~min}$ at $580{ }^{\circ} \mathrm{C}$, which was followed by the $10 \mathrm{~min}$ growth of InP at $585^{\circ} \mathrm{C}$. $[\mathrm{TBP}]$ and $\left[\mathrm{AsH}_{3}\right]$ were the same as above. The actual contents of $\mathrm{P}$ and $\mathrm{As}$ in the grown InAsP layer were not measured, but we expect the content of As in InAsP to be much higher than the supply ratio $\left(\left[\mathrm{AsH}_{3}\right] /\left(\left[\mathrm{AsH}_{3}\right]+[\mathrm{TBP}]\right)=0.36\right.$ in the present experiment $)$ because of the smaller distribution coefficient of $\mathrm{P}^{29)}$ and from our previous results. ${ }^{5)}$ The partial pressure of [TMIn] was $1.04 \times 10^{-6}$ atm, which was determined after the optimization of the InP NW growth conditions.

For characterization, scanning electron microscopy (SEM) and low-temperature PL measurement were carried out. Photon statistics was investigated in the standard Hanbury BrownTwiss measurement setup using superconducting nanowire single-photon detectors. 


\section{Results and discussion}

\subsection{Density control of InP NW array}

Figure 1 summarizes the results of InP-NW growth and its dependence on the period of the NW array. Here, $d_{0}$ was $100 \mathrm{~nm}, T_{\mathrm{G}}$ was $605{ }^{\circ} \mathrm{C}$, the $\mathrm{V} / \mathrm{III}$ ratio was 38.6, and [TMIn] was $2.43 \times 10^{-6} \mathrm{~atm}$. The growth time was $20 \mathrm{~min}$. When $a$ was $1 \mu \mathrm{m}$, a uniform array of NWs was formed, as shown in Fig. 1(a). However, the uniformity deteriorated and some selectively grown InP NWs were anomalously thick when $a$ was $5 \mu \mathrm{m}$, as shown in Fig. 1(b). The deterioration of the uniformity and yield of the NWs with the increase in $a$ is clearly summarized in Fig. 1(c), in which the formation rate of the NWs and their average diameter are plotted as a function of $a$. Although the average diameter $d$ is in the range of 300-350 $\mathrm{nm}$ and nearly independent of $a$, the fluctuation of size increases and the yield decreases as $a$ increases.

As we described in the previous section, the present growth temperature was lower than that used in Ref. 20 and in a more recent study reported in Ref. 30. Actually, the larger $d$, that is, the enhanced lateral growth of the present NWs, suggests that $T_{\mathrm{G}}$ is somewhat lower than the optimum value for obtaining wurtzite NWs with minimal lateral growth, however, we do not consider that this is a major reason for the nonuniformity for large $a$. As shown in the top-right inset of Fig. 1(a), the surface of the side facet of a hexagonal NW is normal to the $\langle\overline{2} 11\rangle$ direction of the InP substrate, which indicates the formation of wurtzite $\mathrm{InP}^{20)}$ surrounded by $\{1 \overline{1} 00\}$ planes. The negligible tapering suggests little inclusion of the zincblende crystal phase. ${ }^{31)}$ The orientation of the hexagon and the degree of tapering are independent of $a$; thus, it is expected that wurtzite InP was formed independent of $a$. This wurtzite phase of InP is formed at a high temperature and a low III-V ratio. In Fig. 1(c), the average height $h$ of the NWs is also plotted as a function of $a$, and within the experimental error, $h$ decreases as $a$ increases. This indicates that the growth is in the synergetic growth mode, ${ }^{27)}$ or the desorption of group III atoms occurred during their diffusion and incorporation into NWs. This situation is discussed in detail in Refs. 20 and 32 in the case of SA-MOVPE, and an important similarity between them is the relatively high growth temperature. Thus, we considered that the temperature is not necessarily lower than that in our previous studies, and the difference in the growth temperature and other conditions is partly due to the difference in the MOVPE system used for the experiment. In fact, the growth of InP NWs at $T_{\mathrm{G}} \geq 635^{\circ} \mathrm{C}$ was carried out but very little growth and short NWs were observed, and the uniformity and yield were not improved. On the other hand, growth at $T_{\mathrm{G}}=575{ }^{\circ} \mathrm{C}$ resulted in the formation of much 

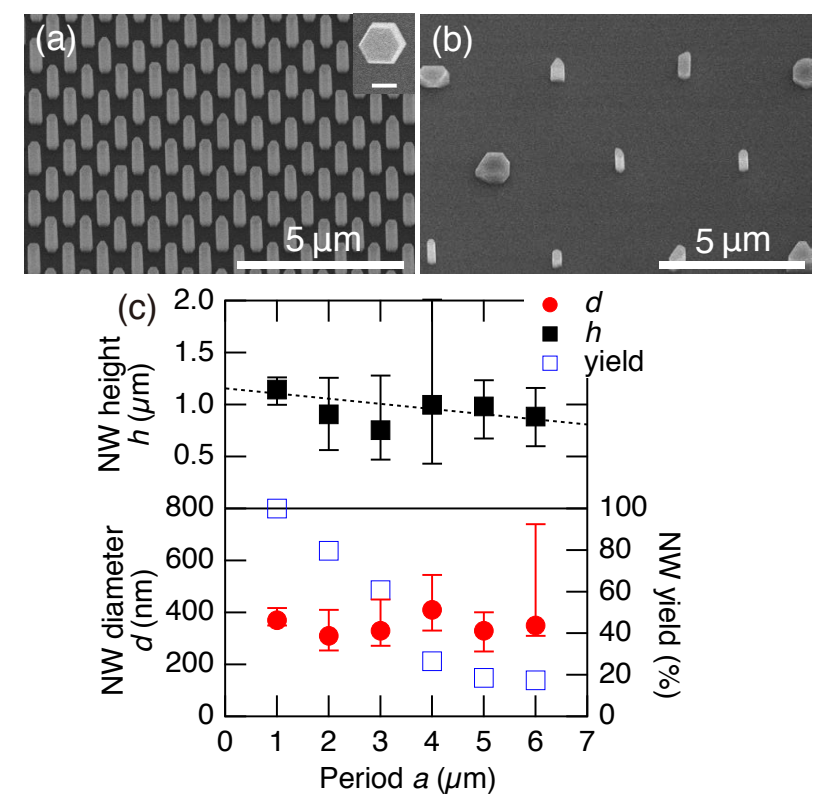

Fig. 1. (Color online) SEM images of InP NWs with (a) $a=1 \mu \mathrm{m}$ and (b) $a=5 \mu \mathrm{m}$. The top-right inset in (a) is a top-view SEM image and the scale bar is $200 \mathrm{~nm}$. (c) Average diameter $d$ and height $h$ of NWs and their formation rate. Bars indicates the maximum and minimum sizes of the NWs measured among 10 to 20 samples. The dotted line is the result of a least-mean-square linear fit for $h$, indicating that the diameter decreases with increasing $a$.

thicker NWs with the zincblende crystal structure, although there was some improvement in the uniformity (see supplementary data for a discussion of growth temperature dependence). Therefore, optimization of growth temperature alone is not very effective for obtaining a uniform array of InP NWs with a sufficiently small NW diameter $d$ and a large period $a$.

As an alternative pathway for optimization, we investigated the dependence of the NW growth on the TMIn supply rate, and the results are summarized in Fig. 2. Here, SEM images of InP NWs with different [TMIn] values and the initial mask openings $d_{0}$ are shown. The diameter $d$ and height $h$ of the NWs obtained in this series of experiments are also summarized in Fig. 3. We can see that reducing [TMIn] and $d_{0}$ resulted in improved size uniformity. For $d_{0}=50 \mathrm{~nm}$ and $\left[\right.$ TMIn] $=1.04 \times 10^{-6}$ atm, we obtained InP NWs with an average $d$ of 67 $\mathrm{nm}$. Note that under these conditions, reasonably uniform arrays of InP NWs were formed for $a$ down to $1 \mu \mathrm{m}$ (see supplementary data). Thus, we can conclude that the density of the NWs was controlled by optimization of the growth conditions.

The growth time of these NWs was fixed at 20 min; thus, the total amount of growth is proportional to [TMIn]. $d, h$, and the volume all increase with [TMIn] independent of $d_{0}$, as shown in Fig. 3. The volume is larger for $d_{0}=100 \mathrm{~nm}$, but $h$ increases and $d$ decreases with 


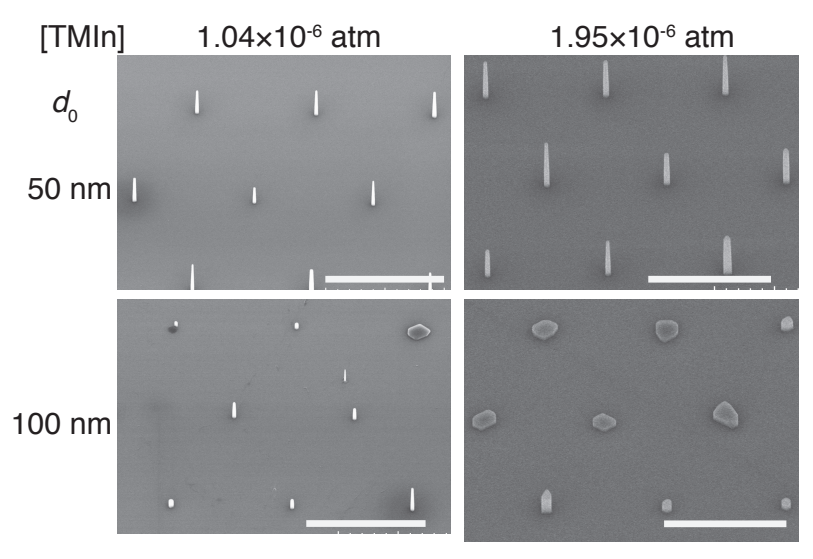

Fig. 2. SEM images of InP NWs showing dependence of NW growth on [TMIn] and opening diameter $d_{0}$. The scale bar is $5 \mu \mathrm{m}$.

decreasing $d_{0}$. The increase in $h$ with the decrease in $d_{0}$ can by explained by the supply of In per unit mask opening area and is often observed in SA-MOVPE growth. ${ }^{32}$ ) The increase in $d$ with $d_{0}$ is obvious. However, it is not clear why reducing In supply rate and decreasing $d_{0}$ result in improved uniformity $d$ and an increase in $h$. However, there is likely to be a simple explanation for this result because the growth proceeds closer to the equilibrium conditions in a low growth rate or low material supply rate conditions and it is generally favorable for obtaining high-quality crystals. In the SA-MOVPE of the NWs, a higher group V supply resulted in the lateral growth of the NWs, as shown in Ref. 20. Thus, increasing the V/III ratio by reducing the group III supply rate would result in enhanced the lateral growth, but this is contradictory to the results of our experiment. The reason for the improvement due to the reduction in $d_{0}$ is also not clear, as a smaller $d_{0}$ resulted in an increase in vertical growth rate. Therefore, the results of the present experiment suggest that the effect of the group III and V materials on the growth of NWs should be considered independently, and the V/III ratio alone is not sufficient to describe the properties of NW growth or the optimization of the growth conditions.

\subsection{Growth and PL characterization of NW-QDs}

Next, InP NWs containing InAsP QDs were grown, which are shown in the SEM images in Fig. 4 We can see that uniform arrays of NWs containing InAsP layers were formed. The NWs were tapered, which is due to the growth at a somewhat lower temperature and the deviation from the ideal growth conditions for wurtzite InP NWs. ${ }^{31)}$ Figure 5 shows the lowtemperature PL spectrum of a single NW-QD. We can see strong emission approximately at 1.49 and $1.44 \mathrm{eV}$, which is attributable to $\mathrm{InP}$ with a mixture of wurtzite and zincblende 


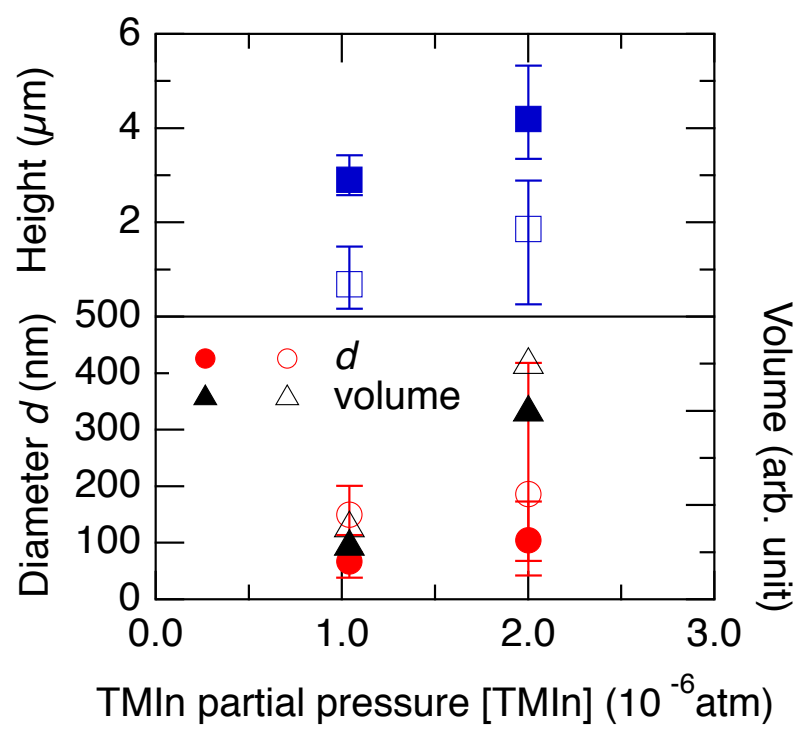

Fig. 3. (Color online) Diameter $d$ and height $h$ of the NWs plotted as a function of [TMIn]. Closed and open symbols indicate the results for $d_{0}=50$ and $100 \mathrm{~nm}$, respectively. The period $a$ of the NW array is $5 \mu \mathrm{m}$.

structures. ${ }^{31)}$ In addition, peaks originating from InAsP were observed, and in this sample two peaks, named X and XX, were observed at approximately $1.325 \mathrm{eV}$. These PL peaks are considered to originate from an exciton and a biexciton in a single QD, which was confirmed by the excitation-dependent measurement of PL (see supplementary data). We measured 95 NWs in the $5-\mu \mathrm{m}$-pitch arrays, and about $90 \%$ of them showed multiple peaks originating from the InAsP layer, and very few showed the signature of the single QDs, as shown by this spectrum. The wavelength of the emission showing multiple peaks extended up to $1.2 \mu \mathrm{m}$, indicating large fluctuations of the composition and thickness of the InAsP layer, which should be prevented for wavelength control. Even in such a case, we were able to confirm the emission of excitons and biexcitons in the QDs from about 10 NWs by a more systematic measurement, one of the results of which is shown in the next section.

In this spectrum of the NW-QD, note that peak XX appears at a higher energy than peak $X$. The energy difference between the emission of excitons and biexcitons was investigated on the basis of their assignment using excitation-intensity-dependent PL, and it was found that almost half of them showed the negative binding of excitons. Because the sample number was not sufficient and the data were scattered, it was not clear whether the negative binding has some dependence on the emission energy of excitons ${ }^{33)}$ (see supplementary data). Nevertheless, the negative binding of excitons originated from the strong confinement of electrons and, in particular, holes, thus, suggesting the formation of a small QD in a NW. 


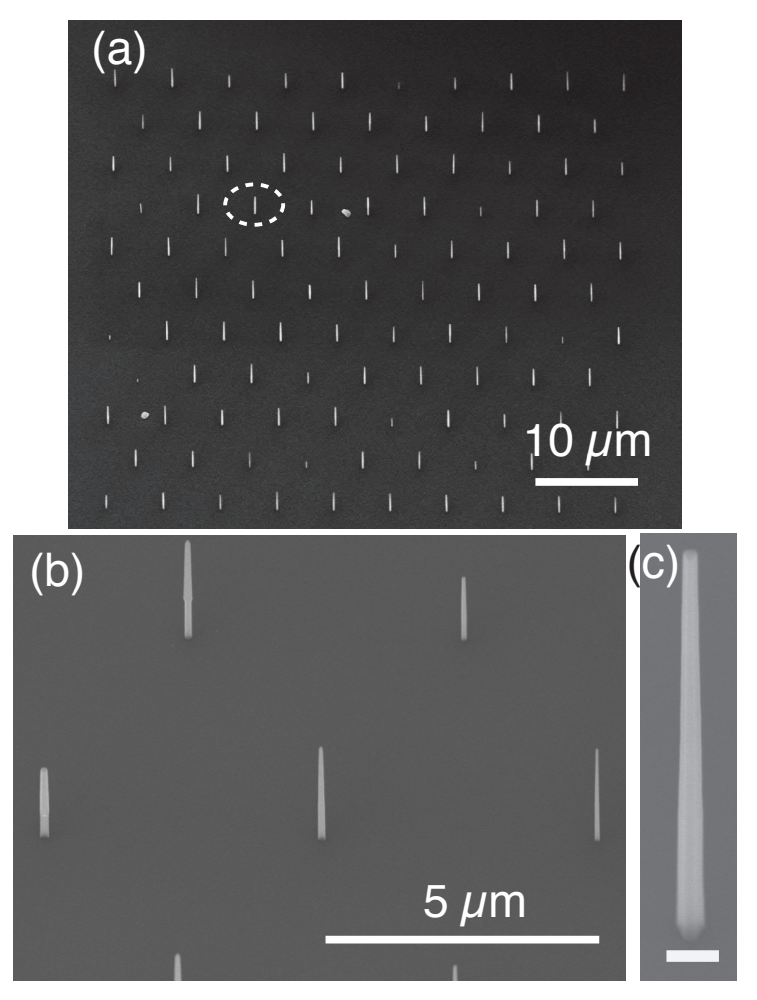

Fig. 4. SEM images of InP/InAsP/InP NW-QD array with period $a=5 \mu \mathrm{m}$. The NW enclosed by the white circle in (a) is the one whose PL results are shown in Fig. 5. The scale bar in (c) is $200 \mathrm{~nm}$.

\subsection{Single-photon correlation measurement}

Finally, we describe the photon statistics of the emission from a NW-QD. The excitation intensity dependence of the PL spectrum of the NW-QD used in the correlation measurement is summarized in Fig. 6. Clear emission due to excitons and biexcitons from the NW-QD was confirmed, respectively, showing linear and quadratic increases in the integrated PL intensity as the excitation intensity increases.

Figures 7 and 8 show typical results of autocorrelation (X-X) and cross-correlation (XXX) measurements under pulsed and cw excitation, respectively. The NW-QD was optically pumped under a barrier excitation condition. The emissions of $\mathrm{X}$ and $\mathrm{XX}$ were spectrally selected by 0.5 -nm-wide band-pass filters after their spatial separation using a 50/50 nonpolarized beam splitter. The photon antibunching behavior in the X-X correlation was clearly observed under both excitation schemes. In the X-X correlation result in Fig. 7, the coincidence count at a zero delay is suppressed and the normalized coincidence with time bins of $4.8 \mathrm{~ns}$ is 0.44 , which is lower than the quantum limit. In the $\mathrm{X}-\mathrm{XX}$ correlation, the time spectrum at around the zero delay is asymmetric and shows an abrupt increase at a negative 


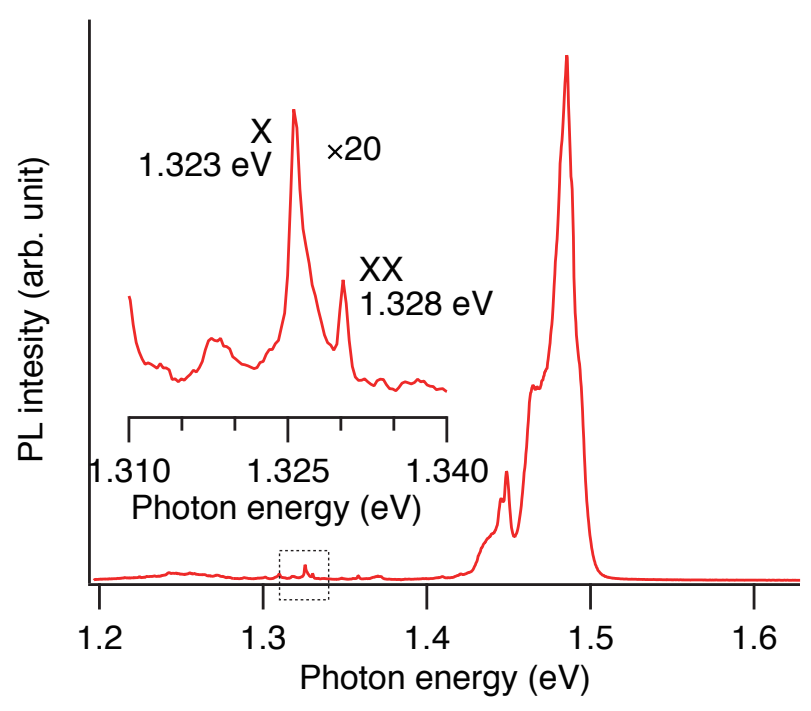

Fig. 5. (Color online) PL spectrum of an InP NW-QD.

delay, which indicates a cascade emission process from the biexciton to exciton state.

Furthermore, cw-like antibunching in the pulse-based X-X correlation was observed, indicating that residual carriers were injected from the InP NW over the timescale of the X-decay of 4.72 ns (see top graph of Fig. 7), which was deduced from the X-pulse excitation laser correlation. Therefore, greater suppression of multiphoton generation was expected; however, insufficient effective time resolution prevented us from determining the $g^{(2)}(0)$ value, which is one of the most important indicators of the purity of single-photon generation.

Therefore, we analyzed the normalized coincidence of $\mathrm{X}$ under the $\mathrm{cw}$ excitation condition (Fig. 8) using a simple three-level model (inset of Fig. 8).

$$
\begin{aligned}
& \frac{d P_{0}}{d t}=\frac{1}{\tau_{\mathrm{X}}} P_{1}(t)-\gamma P_{0}(t) \\
& \frac{d P_{1}}{d t}=\frac{1}{\tau_{\mathrm{XX}}} P_{2}(t)-\left(\frac{1}{\tau_{\mathrm{X}}}+\gamma\right) P_{1}(t)+\gamma P_{0}(t) \\
& \frac{d P_{2}}{d t}=-\frac{1}{\tau_{\mathrm{XX}}} P_{2}(t)+\gamma P_{1}(t)
\end{aligned}
$$

Here, $P_{i}(t)(i=0,1,2)$ represents the probability of a QD containing $i$ excitons at time $t$ ( $i=0$ corresponds to the ground state), $\gamma$ is the pumping rate of $\mathrm{X}$ and $\mathrm{XX}$, and $\tau_{\mathrm{X}}(=4.72 \mathrm{~ns})$ and $\tau_{\mathrm{XX}}(=3.67 \mathrm{~ns})$ are the experimentally deduced decay time constants of an exciton and a biexciton, respectively (see supplementary data). The solid (red) line in Fig. 8(a) shows the fitting results obtained using Eqs. (1a)-(1c), taking into account the multiphoton generation and initial conditions; $P_{0}(0)=1, P_{1}(0)=P_{2}(0)=0 . g^{(2)}(0)$ is evaluated to be $0.200 \pm 0.030$, which is similar to the value obtained in our previous report. ${ }^{24)}$ Figure 8 (b) shows the $\mathrm{X}$ - 
XX correlation under the $\mathrm{cw}$ excitation condition. A typical cascade process can be observed (solid black line). The second-order correlation function of X-XX in the positive $(\tau>0)$ and negative $(\tau \leq 0)$ delay regions is proportional to $P_{1}(\tau)$ and $P_{2}(|\tau|)$ with the initial conditions $P_{1}(0)=1$ and $P_{0}(0)=1$, respectively. The results of fitting based on Eqs. (1a)-(1c), taking into account the multiphoton contribution are shown by the solid (red) line in Fig. 8(b). $\gamma=$ $0.200 \pm 0.009(1 / \mathrm{ns})$ is almost the same as the $\mathrm{X}-\mathrm{X}$ correlation $(0.204 \pm 0.0091 / \mathrm{ns})$. One can see that the photon correlation measurement was well reproduced by the fitting, and $g_{\mathrm{XX}}^{(2)}(0)=0.788 \pm 0.195$ and $g_{\mathrm{X}}^{(2)}(0)=0.183 \pm 0.041$ were obtained. Note that the fittings for the X-X and the X-XX correlations were carried out independently, but the obtained $g^{(2)}(0)$ and $g_{\mathrm{X}}^{(2)}(0)$ were consistent within the experimental and fitting errors.

The $g^{(2)}(0)$ value obtained in the present experiment is comparable to that in our previous report but not as small as the lowest values obtained for InP-based NW-QDs. ${ }^{26)}$ This NW-QD showed multiple peaks originating from the InAsP layer; hence, it is likely that the background emission limits the value of $g^{(2)}(0)$. Thus, to improve and obtain a much smaller $g^{(2)}(0)$, one of the key issues is to suppress unwanted InAsP growth. At present, the formation mechanism of the QDs in our InP NWs has not been clarified, but the formation of QDs on the sidewall as well as on top of the NWs is possibly due to the substitution of P with As. Therefore, further optimization of the growth conditions to guarantee the formation of single QDs on top of NWs is required as well as to control the emission wavelength for use in quantum information and quantum cryptography applications.

\section{Conclusions}

We have succeeded in controlling the density of InP-based NWs by manipulating the growth conditions in SA-MOVPE. The optimization of growth temperature alone was not sufficient to obtain NW arrays with a large pitch and a small NW diameter, and control of the In supply was found to be effective for realizing a uniform NW array. NW-QDs consisting of InP NWs and InAsP quantum dots have also been successfully formed in these density-controlled NWs, and their PL and photon statistics clearly showed the nature of the QDs.

\section{Acknowledgments}

We would like to express our sincere thanks to Professor Takashi Fukui, Professor Katsuhiro Tomioka, Dr. Yoshinori Kohashi, Dr. Hideaki Nakajima, Dr. Shinya Sakita, and Mr. Toshihiro Wada for support in experiments. This work was partly financially supported by a Grant-inAid for Scientific Research from the Japan Society for the Promotion of Science (JSPS). 


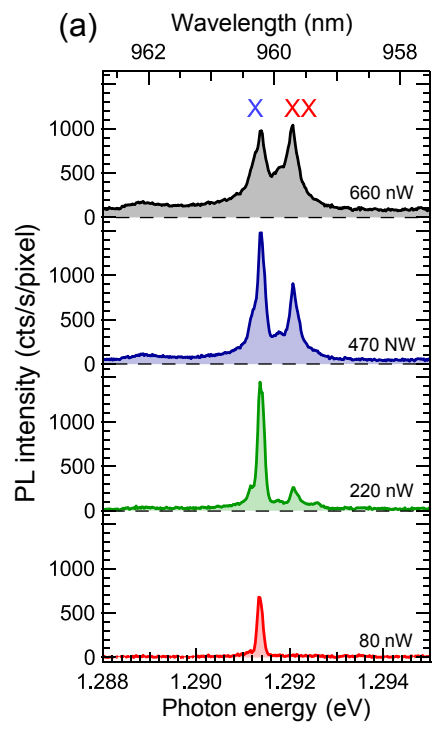

(b)

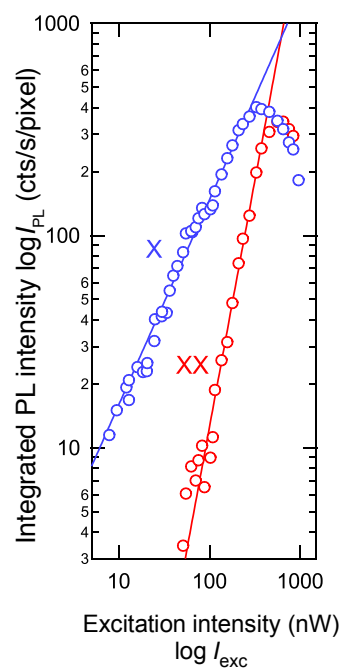

Fig. 6. (Color online) Property of a NW-QD used for the photon-correlation measurement. (a) PL spectra at different excitation intensities. (b) Integrated PL intensity of X and XX peaks plotted as a function of excitation intensity.
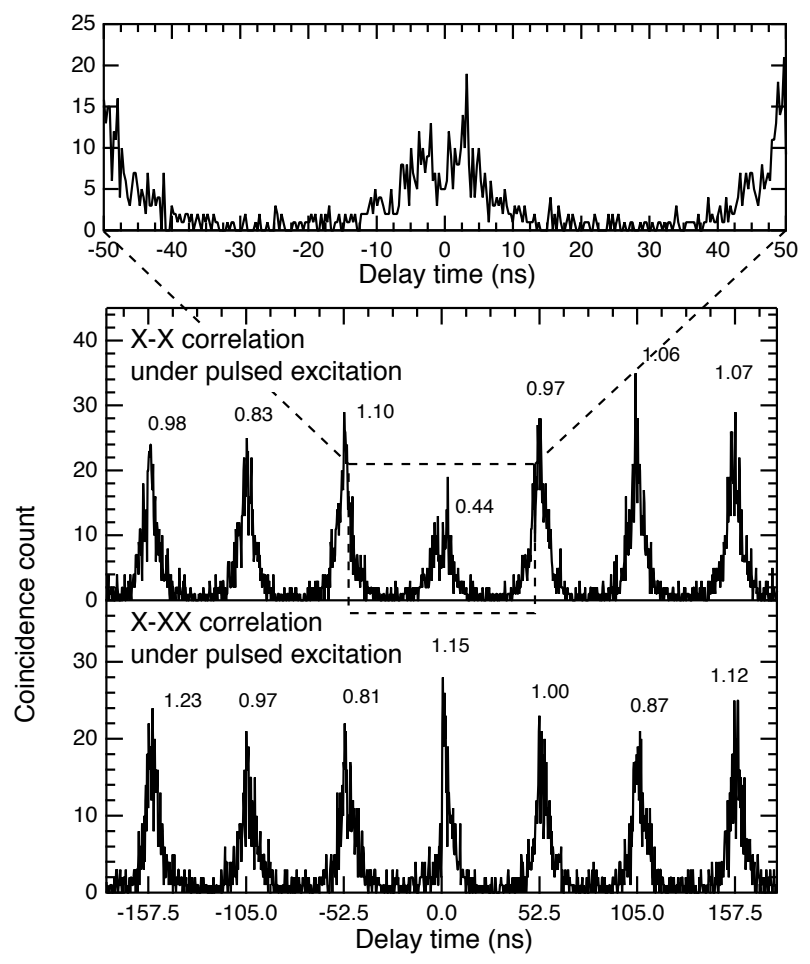

Fig. 7. Results of photon-correlation measurement of a NW-QD under pulsed excitation. (a) X-X correlation and (b) X-XX correlation. Numbers represent normalized counts in $4.8 \mathrm{~ns}$ time bins. 


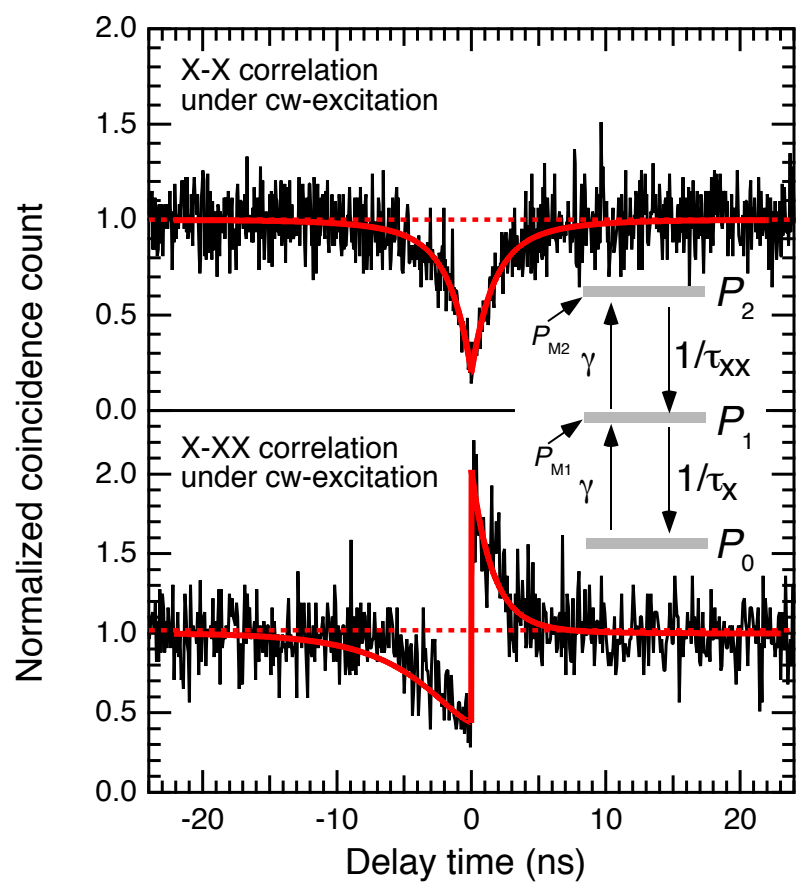

Fig. 8. (Color online) Results of photon-correlation measurement of a NW-QD under cw excitation. Thick solid lines show the results of fitting using a three-level model (inset). 


\section{References}

1) M. Björk, C. Thelander, A. Hansen, L. Jensen, M. Larsson, L. Wallenberg, and L. Samuelson, Nano Lett. 4, 1621 (2004).

2) M. T. Borgström. V. Zwiller, E. Müller, and A. Imamoglu, Nano Lett. 5, 1439 (2005).

3) E. D. Minot, F. Kelkensberg, M. van Kouwen, J. A. van Dam, L. P. Kouwenhoven, V. Zwiller, M. T. Borgström, O. Wunnicke, M. A. Verheijen, and E. P. A. M. Bakkers, Nano Lett. 7, 367 (2007).

4) J. Renard, R. Songmuang, C. Bougerol, B. Daudin, and B. Gayral, Nano Lett. 8, 2092 (2008).

5) Y. Kobayashi, J. Motohisa, K. Tomioka, S. Hara, K. Hiruma, and T. Fukui, Proc. Int. Conf. Indium Phosphide and Related Materials (IPRM 2010), 2010, p.1.

6) J. Tatebayashi, Y. Ota, S. Ishida, M. Nishioka, S. Iwamoto, and Y. Arakawa, Appl. Phys. Lett. 100, 263101 (2012).

7) K. Choi, S. Kako, M. J. Holmes, M. Arita, and Y. Arakawa, Appl. Phys. Lett. 103, 171907 (2013).

8) M. J. Holmes, K. Choi, S. Kako, M. Arita, and Y. Arakawa, Nano Lett. 14, 982 (2014).

9) J. Tatebayashi, S. Kako, J. Ho, Y. Ota, S. Iwamoto, and Y. Arakawa, Nat. Photonics 9, $501(2015)$.

10) S. Maeda, K. Tomioka, S. Hara, and J. Motohisa, Jpn. J. Appl. Phys. 51, 02BN03 (2012).

11) G. Bulgarini, M. Reimer, T. Zehender, M. Hocevar, E. Bakkers, L. P. Kouwenhoven, and V. Zwiller, Appl. Phys. Lett. 100, 121106 (2012).

12) R. Singh and G. Bester, Phys. Rev. Lett. 103, 063601 (2009).

13) A. Dousse, L. Lanco, J. Suffczy'nski, E. Semenova, A. Miard, A. Lemaître, I. Sagnes, C. Roblin, J. Bloch, and P. Senellart, Phys. Rev. Lett. 101, 267404 (2008).

14) N. Somaschi, V. Giesz, L. De Santis, J. C. Loredo, M. P. Almeida, G. Hornecker, S. L. Portalupi, T. Grange, C. Antón, J. Demory, C. Gómez, I. Sagnes, N. D.

Lanzillotti-Kimura, A. Lemaítre, A. Auffeves, A. G. White, L. Lanco, and P. Senellart, Nat. Photonics 10, 340 (2016).

15) X. Duan and C. M. Lieber, Adv. Mater. 12, 298 (2000).

16) S. Bhunia, T. Kawamura, Y. Watanabe, S. Fujikawa, and K. Tokushima, Appl. Phys. Lett. 83, 3371 (2003).

17) U. Krishnamachari, M. Borgstrom, B. J. Ohlsson, N. Panev, L. Samuelson, W. Seifert, 
M. W. Larsson, and L. R. Wallenberg, Appl. Phys. Lett. 85, 2077 (2004).

18) P. Mohan, J. Motohisa, and T. Fukui, Nanotechnology 16, 2903 (2005).

19) M. Mattila, T. Hakkarainen, H. Lipsanen, H. Jiang, and E. I. Kauppinen, Appl. Phys. Lett. 89, 063119 (2006).

20) Y. Kitauchi, Y. Kobayashi, K. Tomioka, S. Hara, K. Hiruma, T. Fukui and J. Motohisa, Nano Lett. 10, 1699 (2010).

21) Q. Gao, D. Saxena, F. Wang, L. Fu, S. Mokkapati, Y. Guo, L. Li, J. Wong-Leung, P. Caroff, H. H. Tan, and C. Jagadish, Nano Lett. 14, 5206 (2014).

22) M. Tchernycheva, G. E. Cirlin, G. Patriarche, L. Travers, V. Zwiller, U. Perinetti, and J.-C. Harmand, Nano Lett. 7, 1500 (2007).

23) D. Dalacu, K. Mnaymneh, J. Lapointe, X. Wu, P. J. Poole, G. Bulgarini, V. Zwiller, and M. E. Reimer, Nano Lett. 12, 5919 (2012).

24) S. N. Dorenbos, H. Sasakura, M. P. van Kouwen, N. Akopian, S. Adachi, N. Namekata, M. Jo, J. Motohisa, Y. Kobayashi, K. Tomioka, T. Fukui, S. Inoue, H. Kumano, C. M. Natarajan, R. H. Hadfield, T. Zijlstra, T. M. Klapwijk, V. Zwiller and I. Suemune, Appl. Phys. Lett. 97, 171106 (2010).

25) H. Sasakura, C. Hermannstädter, S. N. Dorenbos, N. Akopian, M. van Kouwen, J. Motohisa, Y. Kobayashi, H. Kumano, K. Kondo, K. Tomioka, T. Fukui, I. Suemune, and V. Zwiller, Phys. Rev. B 85, 075324 (2012).

26) M. A. M.Versteegh, M. E. Reimer, K. D. Jöns, D. Dalacu, P. J. Poole, A. Gulinatti, A. Giudice, and V. Zwiller, Nat. Commun. 5, 5298 (2014).

27) M. Borgström, G. Immink, B. Ketelaars, R. Algra, and E. Bakkers, Nat. Nanotechnol. 2, 541 (2007).

28) Y. Kohashi, S. Sakita, S. Hara, and J. Motohisa, Appl. Phys. Express 6, 025502 (2013).

29) G. B. Stringfellow, Organometallic Vapor Phase Epitaxy, Theory and Practice (Academic Press, San Diego, CA, 1998), 2nd ed., p. 85.

30) F. Ishizaka, Y. Hiraya, K. Tomioka, and T. Fukui, J. Cryst. Growth 411, 71 (2015).

31) K. Ikejiri, Y. Kitauchi, K. Tomioka, J. Motohisa, and T. Fukui, Nano Lett. 11, 4314 (2011).

32) J. Noborisaka, J. Motohisa, and T. Fukui, Appl. Phys. Lett. 86, 213102 (2005).

33) D. Sarkar, H. van der Meulen, J. Calleja, J. M. Becker, R. J. Haug, and K. Pierz, J. Appl. Phys. 100, 023109 (2008). 